ERRATUM TO :

\title{
CRYSTALLINE DIEUDONNÉ MODULE THEORY VIA FORMAL AND RIGID GEOMETRY
}

\author{
by A. J. de JONG
}

Lemma 7.1.13.1 of [1] is wrong. This was pointed out to the author by Brian Conrad. The only place where Lemma 7.1.13.1 is used in the article is in the construction of the maps $\beta_{n}$ (7.1.13.2). We give a correct construction of $\beta_{n}$ (for A such that $\pi$ is not a zero divisor).

Let $c$ be an integer such that

$$
\pi A \cap I^{n} \subset \pi I^{n-c}
$$

for all $n \geqslant c$. The existence of $c$ is the Artin-Rees lemma. Then for any integer $t \in \mathbf{N}$ we have

$$
\pi^{t} \mathrm{~A} \cap\left(\pi^{t-1} \mathrm{I}^{n}+\pi^{t-2} \mathrm{I}^{2 n}+\ldots+\mathrm{I}^{t n}\right) \subset \pi^{t} \mathrm{I}^{n-c} .
$$

Next, we come to the definition of $\beta_{n}$. Let $n \geqslant c$. Any $a \in \mathrm{A}\left[\mathrm{I}^{n} / \pi\right]$ can be written in the form

$$
a=a_{0}+a_{1} / \pi+\ldots+a_{t} / \pi^{t}, \quad a_{i} \in \mathrm{I}^{i n}
$$

for some $t \in \mathbf{N}$. We simply put $\beta_{n}(a)=a_{0} \bmod \mathrm{I}^{n-c}$. To show that $\beta_{n}$ is well defined, suppose that $a_{0}+a_{1} / \pi+\ldots+a_{t} / \pi^{i}\left(a_{i} \in \mathrm{I}^{i n}\right)$ represents zero in $\mathrm{A}\left[\mathrm{I}^{n} / \pi\right]$. This means that $\pi^{t} a_{0}+\pi^{t-1} a_{1}+\ldots+a_{t}=0$, as $\mathrm{A}$ has no $\pi$-torsion. Thus

$$
\pi^{t} a_{0} \in \pi^{t-1} \mathrm{I}^{n}+\pi^{t-2} \mathrm{I}^{2 n}+\ldots+\mathrm{I}^{t n}
$$

and by the above it follows that $a_{0} \in I^{n-c}$. Hence

$$
\beta_{n}\left(a_{0}+a_{1} / \pi+\ldots+a_{t} / \pi^{t}\right)=a_{0} \bmod I^{n-c}=0
$$

as desired.

[1] A. J. de Jong, Crystalline Dieudonné module theory via formal and rigid geometry, Publ. math. IHES, 82, 5-96 (1995).

Department of Mathematics,

Fine Hall,

Washington Rd

Princeton, NJ 08544-1000

États-Unis. 